\title{
Metodologías Acción participación Cuantitativo aplicado al CC en Nicaragua
}

\section{Methodologies Participation action Quantitative applied to CC in Nicaragu.

\author{
Betanco Ponce, Carmen Abigail; Zúniga-González, Carlos Alberto; \\ Editor académico Prof. Dr. Angel Sol-Sánchez
}

\author{
Carmen Abigail Betanco Ponce \\ abigailbc2010@gmail.com \\ Universidad Nacional Autónoma de Nicaragua, León., \\ Nicaragua \\ iD Carlos Alberto Zúniga-González \\ czuniga@ct.unanleo.edu.ni \\ Universidad Nacional Autónoma de Nicaragua, León., \\ Nicaragua \\ Editor académico Prof. Dr. Angel Sol-Sánchez \\ Colegio de postgraduados, México, México
}

Revista Iberoamericana de Bioeconomía y Cambio Climático

Universidad Nacional Autónoma de Nicaragua, León, Nicaragua ISSN-e: 2410-7980

Periodicidad: Semestral

vol. 2, núm. 4, 2016

czuniga@ct.unanleon.edu.ni

Recepción: 13 Junio 2016

Aprobación: 23 Diciembre 2016

URL: http://portal.amelica.org/ameli/journal/394/3941751015/

DOI: https://doi.org/10.5377/ribcc.v2i4.5937

Autor de correspondencia: abigailbc2010@gmail.com
Resumen: El presente estudio se centró en las metodologías Acción participación aplicado al cambio climático, Se realizó la revisión de la literatura sobre el tema y Los resultados concluyeron que el buen uso de estas técnicas de carácter cualitativo, cuantitativo para obtener información hace de una investigación preexistente, una existente con principios y fundamentos categorizados en la cientificidad y veracidad del campo investigado.

Palabras clave: Metodología, Cuantitativo, Cualitativo.

Abstract: The present study focused on the methodologies Action participation applied to climate change, We conducted the literature review on the subject and The results concluded that the good use of these qualitative, quantitative techniques to obtain information makes a preexisting research, One existing with principles and foundations categorized in the scientificity and veracity of the field investigated.

Keywords: Methodology, Quantitative, Qualitative.

\section{INTRODUCCION}

En el estudio de la metodología aplicada a un campo de investigación es fundamental describir que siempre el empleo de técnicas es primordial para el estudio que se vaya a realizar, en este escrito se describen la metodología, y las técnicas que se emplean en una Investigación Acción Participativa de carácter cualitativocuantitativa, describiéndose cada uno de sus análisis.

\section{NOTAS DE AUTOR}


El análisis de contenido de una información siempre deberá de ser determinada por la técnica que va a hacer empleada, y cada investigador debe de estar sustancialmente definido por las características que su investigación debe de tener al momento de estudiar la situación.

Se determina que dentro de un estudio de investigación, el agente investigador debe de hacer el empelo necesario de las técnicas que ha determinado que se necesitan para que el trabajo sea más efectivo, cabe mencionar que siempre el buen uso de técnicas que van dentro de la metodología, le facilitan que los acontecimientos esperados le sean más rápidos en su comprensión.

\section{METODOLOGÍA}

La metodología es el instrumento del cual depende la técnica que se va implementar para obtener información en una investigación, destacando que siempre debemos de ser cuidadoso al determinar un tipo de estudio aplicando, de manera sustancial las características apropiada para el tipo de estudio que se realizó.

Bogdan y Taylor (1994) opinan que una metodología es "el modo en que enfocamos los problemas y buscamos las respuestas. En las ciencias sociales se aplica a la manera de realizar la investigación”. Cada método debe de ser propio para cada problema que necesitamos dar una respuesta, donde sea necesario.

Basándonos en las aportaciones de Lasso de la Vega (1977), quien dice que la metodología es "un conjunto de procedimientos que sirve de instrumento para alcanzar los fines de la investigación", podemos afirmar que una metodología correcta puede basarse en dos procesos metodológicos complementarios: análisis de contenido y estudio de casos.

Según las aportaciones de Vega se logran discernir que la utilización de instrumentos en una investigación es de gran importancia, se puede decir con certeza que es la medula principal para obtener los resultados ya sean estos de carácter cualitativo o cuantitativo, siempre fundamentados en el análisis previo a la investigación.

Un método fundamental es el análisis de contenido, entendido éste como un método exhaustivo de conocimiento, indispensable para comprender los procesos de comunicación en un área dada.

Considerando siempre que la utilización del método que usted logro establecer sea concreto y coherente en la información que va a descifrar por medio del estudio de la misma, sabiendo que en toda investigación es necesario el buen funcionamiento del método aplicado a dicha investigación.

Usamos el término 'método' cuando nos referimos, (García Ferrando, 1986) a un “componente lógico particular en tanto que se trata de convertir la simple especulación ideológica, filosófica o literaria sobre la sociedad en intentos de comprensión o explicación científica". Este autor, además aporta, entre otras, las siguientes definiciones:

- Lógica de la investigación científica.

- Criterio para el logro del conocimiento científico.

Se comprende que el método debe de ir acompañado de una muy buena investigación científica por el respaldo bibliográfico que debe de llevar toda investigación donde la información debe de ser objetiva, siempre y cuando eso se da en dependencia del caso en estudio, también se describe el criterio de cientificidad que debe de poseer la misma para su validación como una fuente de información confiable ante cualquier lector, ya sea este de carácter social o ambiental

Esta crisis medioambiental a escala mundial, ha originado el surgimiento de la Agroecología, como un enfoque teórico y metodológico que, utilizando varias disciplinas científicas, estudia la actividad agraria desde una perspectiva ecológica y social; para ello, se han de implementar metodologías participativas, basadas en un proceso de interacción creativa dentro de las comunidades rurales, donde el conocimiento local y cientifico se combinan en un diálogo de saberes para la solución de los problemas; la Investigación Acción Participativa ha demostrado su efectivida en tal sentido (Guzmán y Alonso, 2007). 


\section{INVESTIGACIÓN ACCIÓN PARTICIPATIVA.}

La Investigación Acción Participativa (IAP) es un enfoque metodológico que provee de un conjunto de técnicas de investigación-acción útiles para la transición agroecológica (Kindon et al. 2007).

La IAP considera que cualquier proceso de desarrollo que se emprenda estará sesgado, si no integra a los beneficiarios de este proceso como protagonistas del mismo. Su objetivo es generar un conocimiento liberador que parte del conocimiento popular y que explica su realidad globalmente (enfoque sistémico), con el fin de iniciar o consolidar una estrategia de cambio (procesos de transición), paralelamente a un crecimiento del poder político, destinados ambos a alcanzar transformaciones positivas para la comunidad a nivel local; y a niveles superiores en cuanto que es capaz de conectarse con experiencias similares (redes) (Fals Borda, 1991).

Según (Herrera, 2012), definen la investigación -acción como el estudio de un contexto social, para mejorar cualitativamente la acción dentro de ese entorno, de forma crítica. Tiene el doble propósito, de transformación y de generación de conocimiento y compresión. No puede clasificarse como la unión de ambos, sino que es mucho más; es la espiral evolutiva, donde el conocimiento se convierte en acción y ésta a su vez enriquece los saberes, que van a transformar de nuevo a la acción, en una relación dialéctica.

La IAP facilita el diálogo transdisciplinario, a tensión entre teoría y práctica que conduce a un diálogo entre saberes teóricos y saberes prácticos, convirtiendo al investigador en un educador desde el principio freiriano de la "concientización dialógica". (Ortiz y Borjas, 2008)

Por ende se describe la IAP que es un estudio que nos ayuda a definir las técnicas que se van a utilizar en un campo a investigar porque está enmarcada en el contexto social (sociedad) e interdisciplinario, lo cual es diversificado su estudio y que es por este medio que el investigador se convierte en un observador participante porque, está en la acción y el dialogo de una manera persistente con los beneficiarios, desde que comienza el estudio hasta que concluye lo propuesto con un objetivo alcanzado.

\section{TÉCNICAS DE INVESTIGACIÓN.}

Con este término nos referimos a "los procesos específicos a través de los cuales se reúnen y ordenan los datos antes de someterlos a las operaciones lógicas o estadísticas" (Goode y Hatt, 1991)

Las técnicas se refieren a los elementos del método científico, y no deben confundirse con los métodos; sin embargo, su utilización a veces es confusa y llega a hablarse de métodos de investigación cuando en realidad se refiere a técnicas de recogida o análisis de datos. Algunos ejemplos de técnicas son "la entrevista, la encuesta, el grupo de discusión, los experimentos o la escalas de actitudes” (González Ríos, 1997).

Dentro de las técnicas de investigación se suele utilizar la entrevista y la encuesta, también el análisis de contenidos, los cuales sirven como instrumento que pueden medir la información ya sea este carácter Cualitativo o Cuantitativo.

La entrevista: Es un instrumento que sirve en la recolección de datos que se puede hacer por medio de preguntas abiertas a entidades de la comunidad como directores del colegio, enfermeras de la unidad de salud, dirigentes del servicio del agua potable, coordinador o representante de la comunidad, e incluso al alcalde municipal.

La encuesta Es de otro orden donde se realiza a los pobladores que son los mayormente afectados por el problema al cual se están enfrentando, se puede hacer por medio de preguntas abiertas o cerradas, no importa el rango o grado que tenga el encuestado, ya que el fin es de obtener la información que se desea obtener (Barroso, 2015).

El análisis de contenido; Según (Berelson, 1968). Es una herramienta que consta de múltiples utilidades, lo que le confiere un carácter universal, pudiendo servir de método de estudio para infinidad de materias. 
Bardin (1996) define el análisis de contenido como "un conjunto de técnicas de análisis de comunicación tendente a obtener indicadores (cuantitativos o no) por procedimientos sistemáticos y objetivos de descripción del contenido de los mensajes".

Un buen análisis de contenido necesita objetividad y capacidad de medida. Los objetivos fundamentales de un análisis de contenido, según Bardin (1996), son tres:

- Descripción metódica de las características de una comunicación.

- Comprobación de hipótesis para su verificación o rechazo.

- Formulación de explicaciones, sobre contenidos exteriores.

Por su parte, Clemente y Santalla (1991) añaden que el análisis de contenido parte de tres premisas:

1. Comprueba hipótesis mediante deducciones de las peculiaridades del discurso (qué).

2. Comprueba las hipótesis mediante deducciones sobre las causas (quién, por qué).

3. Comprueba esas hipótesis mediante deducciones sobre las consecuencias (para qué).

Dentro de un análisis del contenido es muy importante destacar que los tres principios empleados por Bardin, le hacen ver la coherencia que debe de tener una información al ser obtenida por medio del buen uso de técnicas, asociando lo que describe clemente y Santalla al momento de comprobar la hipótesis con el que quiere saber quién lo desea saber y para que lo desea saber, se comprende que en este momento después del empleo de las técnicas el investigador puede decir con exactitud que si la hipótesis que se había planteado dan los resultados esperados o lo in esperados.

\section{ANALISIS CUALITATIVOS-CUANTIVOS.}

Los partidarios del análisis cualitativo afirman que la supuesta neutralidad y precisión de medida de los datos cuantitativos no pasan de ser una mera afirmación ideológica, resaltando el escaso valor explicativo de varianza aportado por los cuestionarios con fines estadísticos y ridiculizando el abuso esotérico de fórmulas cada vez más cabalísticas para el logro de unas definiciones de los fenómenos sociales progresivamente alejadas de la realidad social (Ruiz, 1996).

En esencia, no se trata de un simple debate metodológico; la raíz del problema reside en la ontología y epistemología de las investigaciones. Es la controversia clásica que enfrenta las aproximaciones positivistas con las interpretativas, la objetividad frente a la subjetividad (Morgan y Smircich, 1980).

La metodología cuantitativa tiene como objetivo discernir las regularidades estadísticas del comportamiento, está orientada hacia el recuento de las frecuencias y medición del alcance del comportamiento que está siendo estudiado (Wildemuth, 1993).

Por lo tanto, aporta información que facilita la generalización de las relaciones. Mientras que la investigación cualitativa tiene como objetivo comprender el mundo social desde el punto de vista de los actores (Wildemuth, 1993) y ayuda a describir el funcionamiento de los sistemas sociales de forma holística, detectando relaciones desconocidas a priori y generando descripciones más completas para facilitar la generalización.

Fidel (1993) enumera las características de la investigación cualitativa: es holística, contextual, inductiva o dialéctica, plural o relativa y está entremezclada con el objeto de la investigación Así, la sustancia de la ciencia resulta ser cualitativa y cultural; no es la sola medición estadística, sino la comprensión de las realidades" (Fals Borda, 1981).

En los estudios cualitativos, los investigadores siguen un diseño de la investigación flexible. Comienzan sus estudios con interrogantes formuladas vagamente Álvarez-Gayou, J. L. (2003). “En los estudios cualitativos el tamaño de muestra no es importante desde una perspectiva probabilística, pues el interés del investigador no es generalizar los resultados de su estudio a una población más amplia" (Hernández, Fernández y Baptista, 2010). 
Considerando que el análisis cuantitativo y cualitativo es fundamental cuando la investigación es documentación bibliográfica o por terminología, se destaca que una de este tipo de análisis determina la información de la otra, porque uno lo interpreta en una determinación numérica y la otra lo asigna con el conocimiento que describió, y pasan de ser de objetos no medibles a medibles.

Rodriguez (2011) nos argumenta que la investigación forma parte del conocimiento humano en general y por tanto el conocimiento se ha definido como un proceso en el cual se realcionan un sujeto cognoscente (quien conoce) con un objeto de conocimiento (aquello que se conoce) lo que da como resultado un producto mental nuevo, llamado conocimiento. Así, el mismo término designa al proceso y al resultado de dicho proceso; es decir, llamamos conocimiento tanto a la operación subjetiva que lo produce, como al mismo producto.

Zúniga et. al (2016) describe que algunas estrategias cualitativas de recogida de información son la observación del participante, y las entrevistas cualitativas, documentos oficiales, revistas, documentos internos, dossiers, estatutos, expedientes personales, diarios, cartas, autobiográficas. Es importante aclarar que cada técnica tiene su guía de elaboración y que va en dependencia del tema de investigación.

Se logra comprender que toda investigación donde quiera que este, el investigador siempre busca una respuesta a un fenómeno que logra percibir y que él considera que puede tener una respuesta, solo que hay que emplear métodos y técnicas para que el estudio de mismo sea más fácil y en un corto tiempo.

\section{Conclusiones.}

La metodología ha combinado diferentes técnicas de investigación dentro de un proceso participativo, que la ha llevado a hacerse más elocuente, donde cada técnica tiene su propia manera de ser aplicada, de esta forma ayudan a que sus avances, en el estudio de las ciencias sea este más emotivo ya sean estas teóricas o experimentales.

La IAP es la pauta que genera las distintas técnicas en el momento de realizar una investigación, y que su estudio es fundamental para determinar lo que se pueden implementar al momento de realizar el ejercicio como tal, por ende el estudio del, CC, se pude determinar los resultados específicos por medio de la encuesta y la entrevista, o análisis de contenido donde hay parámetros medible y no medibles para la obtención de datos de investigación.

El buen uso de instrumentos en una investigación nos conlleva a resultados exactos y satisfactorios en el momento en que se requiere de datos que lo lleven a dar una respuesta a la problemática planteada, que cada investigador se propone cuando estudia una situación-problema en una zona determinada.

\section{Referencias Bibliográficas}

Álvarez-Gayou, J. L. (2003). Cómo hacer investigación cualitativa. Fundamentos y metodología. Colección Paidós Educador. México: Paidós Mexicana.

Barroso, C. (marzo de 2015). Los instrumentos de la investigación acción participativa. Recuperado el 10 de marzo de 2015, de www.ull.es: http://www.ctinobar.webs.ull.es/1docencia/Cambio\%20Social

Bardin, L. (1996): El análisis de contenido. Madrid, Akal.

Berelson, B. (1968): Content analisis in communications research. Nueva York, Free press.

Bogdan, R. y Tayor, S. J. (1994): Introducción a los métodos cualitativos de investigación. Barcelona, Paidós.

Clemente, M. y Santalla, Z. (1991): El documento persuasivo. Análisis de contenido y publicidad. Bilbao, Deusto.

Fidel, R. (1993): "Qualitative methods in information retrieval research”, en Libraryand Information Science Research n. 15, pp. 219-247.

García Ferrando, M. (1986): Sobre el método. Madrid, CIS 
Guzmán, G. y Alonso, A. (2007). La investigación participativa en agroecología: una herramienta para el desarrollo sustentable. Revista Ecosistemas 16 (1), España, 24-36 p. http://www.revistaecosistemas.net/articulo.asp? $\mathrm{Id}=46.6$.

Morgan, G., \& Smircich, L. (1980). The case for qualitative research. Academy of management review, 5(4), 491-500.

Lasso de la Vega, J. (1977). Cómo se hace una tesis doctoral. Cómo se hace una tesis doctoral. Técnicas, normas y sistemas para la práctica de la investigación científica y técnica y la formación continuada. Madrid, Fundación Universitaria Española

Fals Borda, O. (1991). Algunos ingredientes básicos. In: Acción y Conocimiento. Como romper el monopolio con investigación-acción participativa, 7-19 Santafé de Bogotá: CINEP.

González Río, M. J. (1997). Metodología de la investigación social. Técnicas de recolección de datos. Aguaclara, Madrid, Fundación Universitaria Española.

Goode, W. J., \& Han, P. K. (1970). Métodos de investigación social México. Trillas.

Herrera, H. E. R. (2012) Seminario Taller de investigación. Diplomado en Gestión de Riesgo y Cambio Climático. Centro de Formación y Capacitación Permanente para la gestión integral de Riesgos y Desastres. UNAN Managua - FAREM Estelí

Hernández, R., Fernández, C. y Baptista, P. (2010). Metodología de la Investigación. Cuarta edición, editorial Mc Graw Hill, México, D. F., p.613.

Kindon, S., Pain, R. \& Kesby, M. (2007). Participatory Action Research Approaches and Methods. Routledge Series in Human Geography. Oxon: Routledge

Ortiz, M., \& Borjas, B. (2008). La Investigación Acción Participativa: aporte de Fals Borda a la educación popular. Espacio abierto, 17(4), 615-627.

Rodríguez, J. M. (2011). Métodos de investigación cualitativa. Revista de Investigación Silogismo, 1(08).

Wildemuth, B. (1993): “Post-positivist research: two examples of methodological pluralism”, Library Quarterly, 63(4), 450-468.

Zúniga-González, C. A., Jarquín-Saez, M. R., Martinez-Andrades, E., \& Rivas, J. A. (2016). Investigación acción participativa: Un enfoque de generación del conocimiento. Revista Iberoamericana de Bioeconomia y Cambio Climático, 2(1), 218-224. 